\title{
Immunoregulatory properties of serum from patients with different stages of syphilis
}

\author{
J J THOMPSON, R J MANGI, R LEE, AND J M DWYER \\ From the Division of Immunology, Department of Medicine, Yale University School of Medicine, \\ New Haven, Connecticut, USA
}

SUMMARY The response of lymphocytes from 17 patients with primary, secondary, and tertiary syphilis to phytohaemagglutinin (PHA) and to allogeneic lymphocytes was normal in heterologous serum; however, the responsiveness of cells from some patients with primary and secondary disease was significantly reduced in the presence of autologous serum. As cells from healthy controls invariably responded better to these stimuli in autologous serum, the sera from 81 patients with syphilis were screened for immunosuppressive properties. Sera from 25 primary, 32 secondary, two tertiary, six congenital, and 16 latent cases of syphilis were examined for their ability to reduce the responsiveness of normal cells to PHA. These experiments were performed with test sera as the sole source of serum for the cultures or with test sera added to cultures containing optimally supportive amounts of pooled human plasma.

Stimulation of normal cells from one control in human plasma and $20 \%$ test serum showed that only in sera from congenital cases of syphilis was the mean response significantly different from the response seen in control sera; a significant increase in the response to stimulation occurred. The range of response to PHA with sera from cases of primary and secondary syphilis was wider than with normal sera. Sera from five $(20 \%)$ cases of primary and $14(44 \%)$ cases of secondary syphilis appeared to be immunosuppressive. When retested on another sample of normal cells, these sera were consistently immunosuppressive even in the presence of $15 \%$ pooled human plasma. Thus, in early syphilis antigenic stimulation may result in the release from suppressor cells of non-specific immunoregulators of cell-mediated immunity. Such phenomena may be a prelude to the development of tolerance to treponemal antigens. In congenital syphilis the development of suppressor cells may be impaired, resulting in the apparent immunostimulatory properties of serum from such cases.

\section{Introduction}

Many biological systems are regulated by balancing opposing forces. Conclusive evidence is now available to extend this principle to the immune response. ${ }^{1}$ Reaction to foreign antigens represents, at any given moment, a balance between immunoregulatory and suppressive forces, provided mainly by a subset of thymus-derived lymphocytes and effector mechanisms that produce inflammatory responses by antibody production or cell-mediated immunity (CMI). ${ }^{23}$ Although triggered by specific antigen, suppressor influences are frequently nonspecific and can thus produce generalised

Address for reprints: Dr J M Dwyer, Yale University School of Medicine, 333 Cedar Street, New Haven, Connecticut 06510, USA

Received for publication 13 August 1979 immunosuppression. In a number of granulomatous diseases and parasitic infections interaction between the host's immune system and the causative antigen(s) produces a response that favours suppression rather than effector mechanisms. ${ }^{4-6}$ This gives an invading organism capable of preferentially activating suppressor machanisms definite survival advantages. The immunological response to the micro-organism causing syphilis may be similar. An adequate cell-mediated immune response to Treponema pallidum may be hampered by specific activation by the organism of suppressor mechanisms. ${ }^{78}$ Consistent with this concept are studies which suggest that in active disease both delayed hypersensitivity and in-vitro lymphocyte responsiveness to $T$ pallidum are best seen after treatment. ${ }^{9-11}$ 
The suppression of lymphocyte responses to mitogens and non-treponemal antigens in syphilis seems definite, although conflicting data exist on the degree and time of appearance of this non-specific immunosuppression. In two studies lymphocyte responsiveness to phytohaemagglutinin (PHA), a $\mathrm{T}$-cell mitogen, was not reduced at any stage of the disease. ${ }^{12}{ }^{13}$ However, most studies show suggestive if inconclusive evidence for altered CMI in primary, secondary, and congenital syphilis. In a retrospective study of the histology from the spleen of children who died of congenital syphilis before the age of 3 numbers of lymphocytes in $\mathrm{T}$-cell areas were decreased. ${ }^{8}$ Inguinal node biopsies from some untreated patients with secondary disease showed a similar depletion. ${ }^{8}$ Using cells from subjects with primary, secondary, tertiary, and latent syphilis, other workers ${ }^{89}$ concluded that the lymphocytes from patients with primary and secondary disease responded poorly when stimulated by PHA in the presence of their own plasma. Furthermore, the plasma from patients with secondary disease could reduce the response to PHA of cells from a healthy subject. Some authors have suggested that the immunosuppressive factors could only be demonstrated with plasma.

If active suppression of the host's response to $T$ pallidum is accomplished by antigen-specific activation that in turn leads to the production of nonantigen specific suppressor lymphokines, these humoral agents will be concentrated at infection sites. Thus, remote from such sites biological fluids (for example, plasma) may contain diluted lymphokines capable of inducing a weak but measurable non-specific suppression of lymphocyte function.

We have examined plasma samples from patients with syphilis for immunosuppressive properties, but previous studies from this laboratory of a similar question in sarcoidosis ${ }^{14}$ have shown that it is difficult to prove that serum factors are actively immunosuppressive rather than passively nonsupportive. In the present study stimulation by PHA of lymphocytes from one healthy subject in many different but normal sera produced an extremely wide variation (100-fold) in the degree of stimulation measured. We therefore consider that biologically significant suppressors in sera should be looked for by adding test sera to cultures already with optimal supportive conditions incorporating pooled human plasma. We have used this approach in an attempt to clarify and extend the significance of the previous studies of serum modifiers of cell-mediated immunity in syphilis. Furthermore, we have looked at the immunocompetence of thoroughly washed lymphocytes from patients with syphilis by stimulating such cells with PHA and allogeneic lymphocytes (mixed lymphocyte reaction, MLR) in media containing normal sera.

\section{Materials and methods}

\section{STUDY POPULATION}

\section{Cells}

To assess cellular immune competence in syphilis, cells from 17 patients and 10 controls were studied. Five primary, 11 secondary, and one tertiary case of syphilis were evaluated. The patients attended either the Mothaven or Morisanea Clinics of the New York City Health Department or the Yale-New Haven Hospital. The 10 controls were laboratory personnel with negative results to the Venereal Disease Research Laboratory (VDRL) test. Only patients with ulcers giving positive results on darkground microscopy were considered to be cases of primary syphilis. Clinical signs of secondary disease, consisting of a rash as well as a VDRL titre consistent with active secondary disease, were required for a diagnosis of secondary syphilis. The one late case had untreated tabes dorsalis. The clinical history, laboratory investigations, treatment, age, sex, and race of each case were recorded.

\section{Sera}

To assess humoral immunoregulators in syphilis, sera from 81 patients and 33 controls were examined for the presence of immunosuppressive factors. Sera from 25 primary, 32 secondary, two late, six early congenital, and 16 latent cases of syphilis were studied. Most of these sera, as well as the age, sex, race, serological results, and history of the patients from whom they were drawn, were supplied by the Communicable Disease Centre (Atlanta, Georgia, USA).

\section{Lymphocyte cultures}

Heparinised blood was allowed to sediment for two hours at $37^{\circ} \mathrm{C}$. The leucocyte-rich plasma was centrifuged twice at $1800 \mathrm{rev} / \mathrm{min}$ at $4^{\circ} \mathrm{C}$ for 10 minutes. The pellet was resuspended in $2 \mathrm{ml}$ of Roswell Park Memorial Institute medium 1640 (RPMI 1640) containing penicillin, streptomycin, L-glutamine, and heparin. ${ }^{14}$ The cell concentration was adjusted to $4 \times 10^{6}$ per $\mathrm{ml}$, and $0.05 \mathrm{ml}$ $\left(0.2 \times 10^{6}\right.$ cells) was placed in a microtissue culture well. For studies of cellular competence, stimulation with PHA and allogeneic lymphocytes (MLR) were performed with the cells in both pooled human plasma (PHP) $15 \%$ and $15 \%$ PHP with $20 \%$ autologous plasma (AP) added. To assess the effect of serum from patients with syphilis on normal cells, the latter were stimulated with PHA in media 
containing either $20 \%$ test serum or $15 \%$ normal PHP as well as $20 \%$ test serum. These decisions were taken after initial studies (reported below) showed wide variations in the responsiveness of normal cells in various normal sera. Cells were stimulated by an optimal concentration of PHA ( $1 / 250$ stock solution) or a mixture of inactivated lymphocytes containing equal numbers of cells from four subjects (total $0 \cdot 4 \times 10^{6}$ ). Cultures using PHA were pulsed with tritiated thymidine and terminated after three days whereas the MLRs were pulsed and terminated after seven days. Samples were harvested using a multiple automated sample harvester and thymidine uptake measured by a standard method.$^{14}$ Mean values were obtained from quadruplicate studies. Increases in absolute counts per minute (cpm) after stimulation were calculated by subtracting from the counts obtained from stimulated cultures the baseline $\mathrm{cpm}$ emitted from unstimulated cells.

\section{STATISTICAL METHODS}

Statistical significance for the differences in lymphocyte responsiveness was obtained by comparing mean values and the standard error of the mean (SEM) using Student's $t$ test.

\section{Results}

EFFECTS OF NORMAL PLASMA ON RESPONSE OF NORMAL LYMPHOCYTES TO PHA

The results of studies in which cell cultures from the one individual were established on one day and stimulated with one dose of PHA are given in fig 1. The cultural conditions were identical except for the plasma added to the culture media. Most striking is the effect of heterologous plasma (HP) on responsiveness to PHA. Ten different normal plasma samples produced videly scattered results with a mean \pm 1 standard deviation (SD) for absolute cpm of $27753 \pm 14132$. This can be compared to the mean $\mathrm{cpm} \pm 1 \mathrm{SD}$ of 10 cultures containing the same cells cultured in autologous plasma (AP), $37675 \pm 2875$, or 10 samples of pooled human plasma (at least four separate samples pooled) of $36280 \pm 3864$. Clearly, some of the normal HP samples looked immunosuppressive but were simply non-supportive. In a clinical study, four of the 10 samples would have been considered abnormal. By culturing these same cells in $15 \%$ PHP and adding $20 \%$ AP or HP, normal stimulation with an acceptable standard deviation was obtained. For this reason we considered that biologically significant suppression of lymphocyte responsiveness by syphilitic plasma should be looked for in cultures already optimally supported by pooled human plasma.

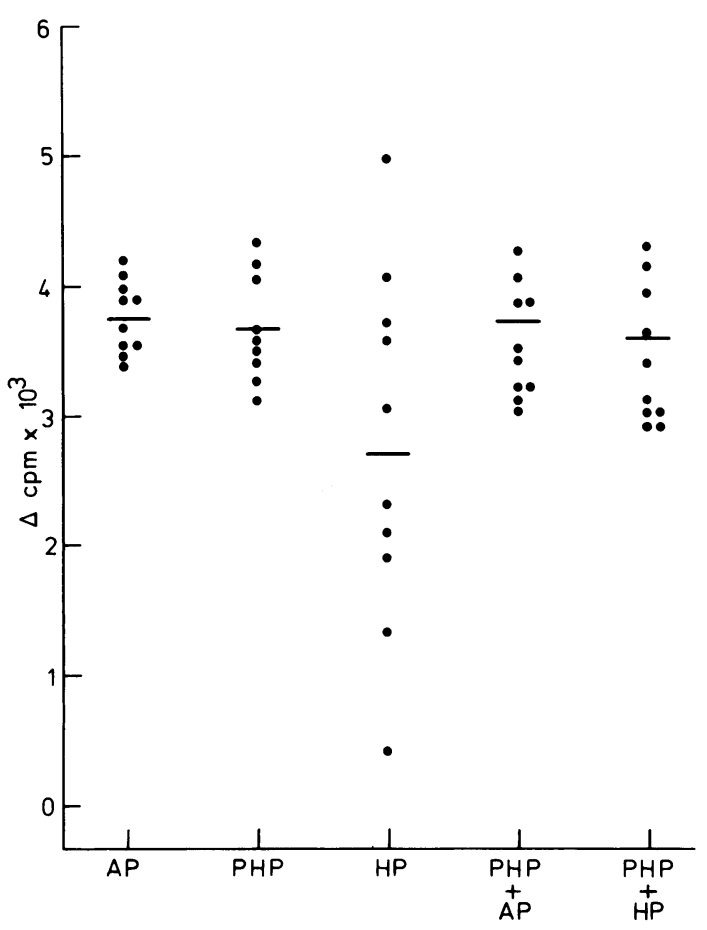

FIG 1 Results of 10 identical cultures established with cells from one individual in his own plasma (15\%). The variation in DNA synthesis so obtained (which is inherent in the methodology used) was compared to the variation obtained when different plasma samples were substituted for AP. The same cells were stimulated in 10 different samples of PHP (15\%), HP 15\%, 15\% PHP and $20 \%$ $A P$, and $15 \%$ PHP and $20 \% \mathrm{HP}$.

\section{STIMULATION OF LYMPHOCYTES FROM SYPHILITIC PATIENTS \\ Phytohatmagglutinin}

The data obtained from stimulating normal cells and cells from patients with primary, secondary, and tertiary syphilis with PHA in $15 \%$ PHP, or in $15 \%$ PHP and 20\% AP, are shown in fig 2 together with the mean increase in absolute cpm and a range of $\mathrm{cpm}$ representing $2 \mathrm{SD}$. The response of normal control cells to PHA was better in media containing AP $(P<0 \cdot 001)$; the same was true for most patients with syphilis. However, the cells from three primary and four secondary cases of syphilis responded better in PHP suggesting a positive inhibitory effect of their serum on these cells. There was no significant difference between the baseline counts from unstimulated cultures in either PHP or AP. Although the mean response to PHA of the cells from patients with secondary syphilis was lower than that of the corresponding control group, the 


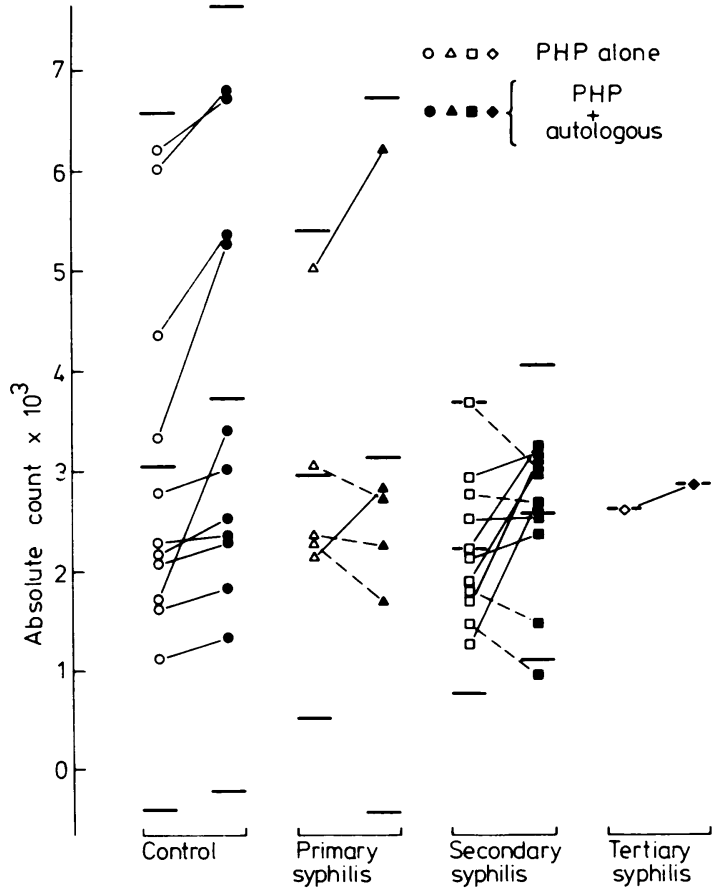

FIG 2 Results of stimulation of normal cells and cells from patients with syphilis with $\mathrm{PHA}$ in either $15 \% \mathrm{PHP}$ or $15 \%$ PHP and 20\% AP. The mean increase in cpm \pm 2 $S D$ is given for each group.

differences were not statistically significant. Thus, no evidence was found to suggest that on group analysis the cells from patients with primary, secondary, or tertiary disease in the absence of AP were less responsive to PHA than normal cells (fig 2).

\section{Pooled irradiated lymphocytes}

The results obtained using pooled irradiated lymphocytes paralleled those found with PHA (fig 3). Again, normal cells responded better to allogeneic lymphocytes when AP was present $(P<0 \cdot 05)$. Cells from one of the four patients with primary syphilis responded better in PHP cultures. Cells from six of 11 subjects with secondary disease responded less well to allogeneic lymphocytes in the presence of AP. Despite this clear evidence of some immunosuppressive factor in the plasma of some patients with syphilis, no intrinsic defect in the ability of the cells to respond to allogeneic lymphocytes was noted.

\section{RESPONSE OF NORMAL CELLS TO PHA}

\section{Effect of syphilitic sera}

Initially, the response of one control's normal cells to PHA was studied in 15\% PHP containing $20 \%$ test

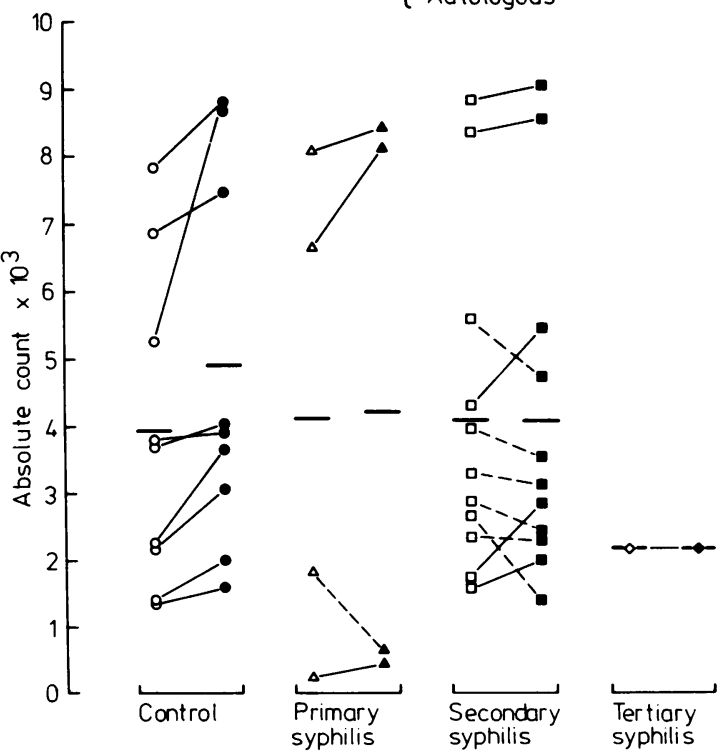

FIG 3 Results of stimulation of cells from controls and patients with syphilis with allogeneic cells in either PHP or PHP and $A P$.

serum. Thirty-two control sera and sera from 24 primary, 26 secondary, six congenital, and 13 latent cases of syphilis were tested (first series). All cultures were set up on the same day thus avoiding the inherent variation in the response of cells from one individual at different times. Comparison of the mean response to PHA and the standard deviation observed in each group by unpaired $t$ tests showed that only in the presence of the sera from congenital cases was there a significant difference. Compared with the response of normal cells in control sera and PHP, the same cells in the presence of PHP and sera from congenital cases showed a significant increase $(P<0.001)$ in their response to stimulation (fig 4). The response to PHA of the cells from this control subject did not vary significantly when a range of $10-40 \%$ PHP was tested to ensure that the presence of $35 \%$ plasma/serum in the cultures was not in itself a stimulating or suppressive factor. As shown in fig 4 , the mean response of normal cells in sera from primary or secondary cases did not differ significantly from that when control sera were used. However, the range of response was different. With sera from four cases of secondary disease and one case of primary disease, the stimulation observed fell more than 2 SD from the mean value observed with 


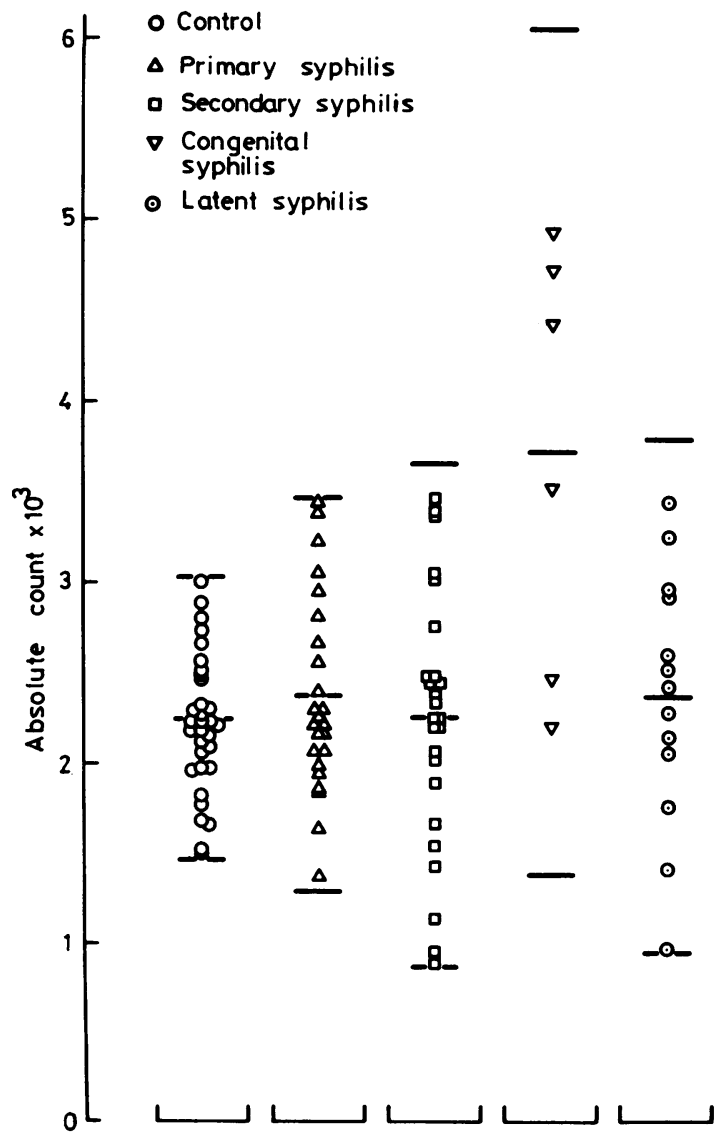

FIG 4 Results of stimulation of lymphocytes from one control with PHA in $15 \%$ PHP containing $20 \%$ test sera.

control sera. Because of this observation, and the suggestions from studies using the patients' cells that some sera from cases of primary or secondary disease were immunosuppressive, a second study was performed comparing the cellular response of normal cells to PHA in test sera alone and in test sera with PHP.

\section{Effect of test sera with and without PHP}

The effects of sera from 32 controls and 24 primary, 27 secondary, two late, six congenital, and 16 latent cases of syphilis on the response of normal cells to PHA were evaluated. The test sera were used either as the sole serum source $(20 \%)$ (fig 5 ) or together with $15 \%$ PHP (fig 6). One healthy control supplied all the cells for this study and cell cultures were set up on the same day. With the exception of cultures containing sera from cases of congenital syphilis, the mean of the stimulation observed was higher when

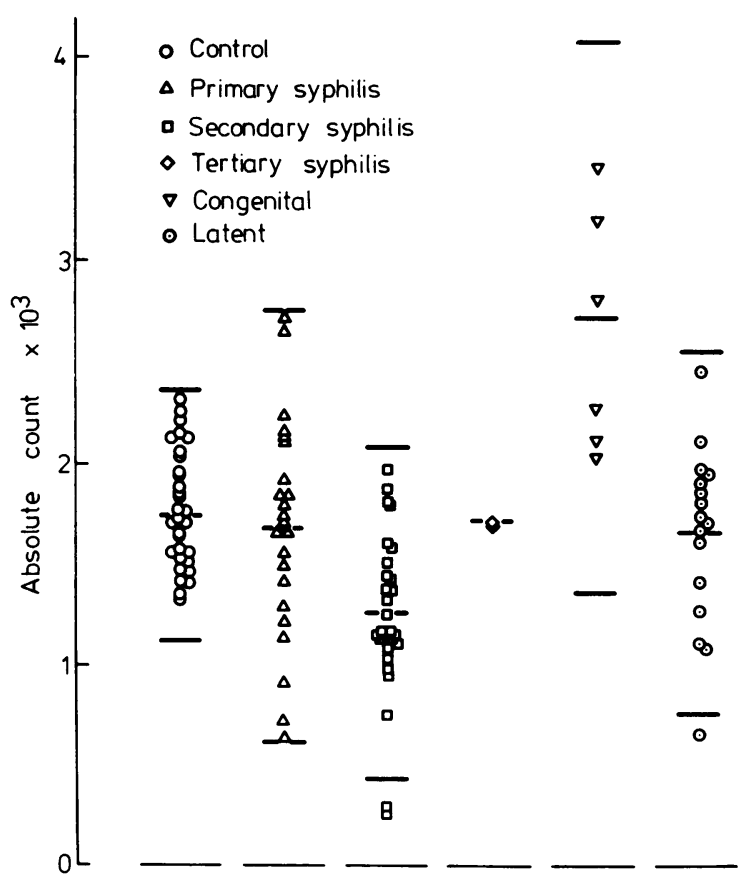

FIG 5 Results of stimulation of lymphocytes from one control with PHA in $20 \%$ test sera without PHP.

PHP was added to test sera, even in the studies using normal sera. Clearly, PHP is able to dilute the immunosuppressive effect that some normal and test sera exert on normal cells (fig 6). Comparison of the mean and standard deviation of the stimulation observed in studies where PHP was added to test sera again showed no significant difference between the response of the normal cells to PHA in control sera or sera from cases of primary, secondary, or latent syphilis. Again, the exception was in sera from congenital cases, which increased the degree of stimulation. No significant difference between the mean stimulation values obtained with control sera alone and with sera from primary, late, or latent cases of syphilis alone was noted. However, when normal cells were cultured in serum from secondary cases of syphilis without added PHP the response to PHA was significantly reduced $(P<0.05)$ (fig 5$)$. The use of sera from congenital cases of syphilis without PHP was associated with significantly better responses to PHA than when normal sera were used $(\mathrm{P}<0 \cdot 001)$. A comparison of the range of response in the different conditions shows a wider scatter with sera from cases of primary and secondary syphilis than with normal sera. 


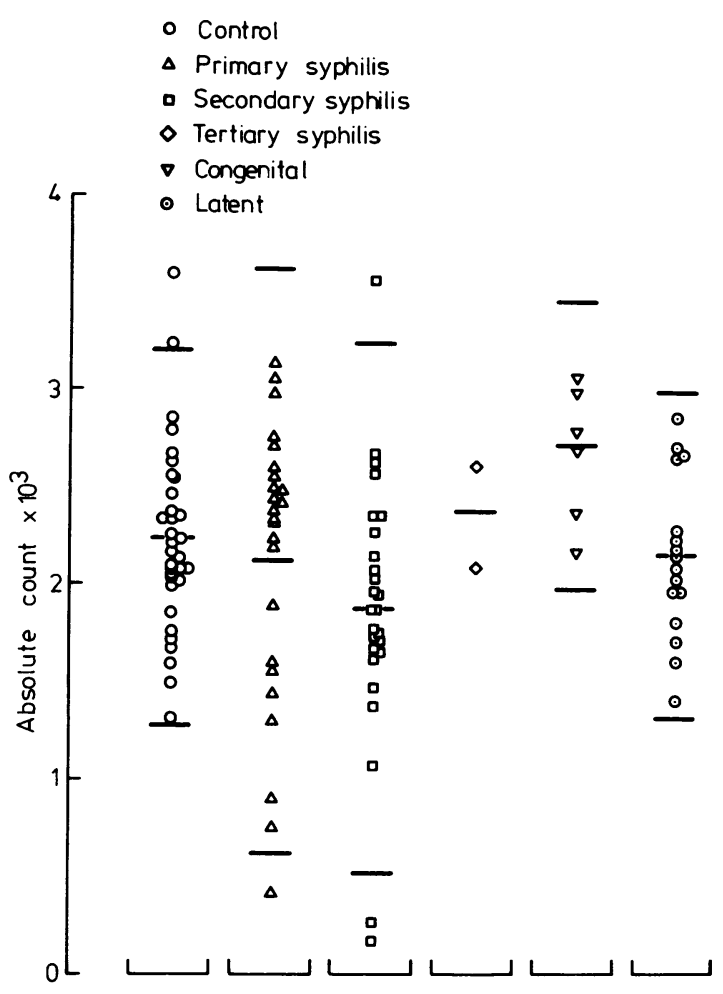

FIG 6 Results of stimulation of lymphocytes from one control with PHA in test sera containing 15\% PHP.

\section{REPRODUCIBLE IMMUNOSUPPRESSION BY SELECTED SYPHILITIC SERA}

Sera from five cases of primary and 14 cases of secondary syphilis, previously associated with levels of stimulation of normal cells that fell 2 SD below the mean value observed when the same cells were cultured in normal sera, were retested together with 13 control sera. Normal cells from a healthy control, not previously used in these experiments, were stimulated with PHA in the presence of $20 \%$ test sera and $15 \%$ PHP. With these selected sera the presence of PHP did not dilute their immunosuppressive effect. When the mean increase in absolute counts emitted by the normal cells after PHA stimulation in normal sera are compared with the values obtained with sera from primary and secondary cases of syphilis a significant reduction $(P<0.05$ and $P<0.001$ respectively) is observed in the presence of these latter sera. As the cells had an optimal nutritional supply provided by the PHP these selected sera appear to be significantly and reproducibly immunosuppressive.

\section{Discussion}

Shortly after the organism causing syphilis was discovered in $1905,{ }^{15}$ studies to determine the degree of immunity from reinfection that followed primary exposure to $T$ pallidum began. These studies have been inconclusive. Perhaps as a result of poor antigenic preparations, numerous attempts to elicit delayed hypersensitivity (DH) reactions to treponemal antigens have provided inconsistent data. ${ }^{16-21}$ The available -data suggest that $\mathrm{DH}$ to treponemal antigens is absent in the primary and secondary stages of the disease whereas some $\mathrm{DH}$ is occasionally seen in later stages of the disease and in congenital disease.

Better evidence for the development of immunity to syphilis came from a study in which virulent organisms were inoculated into volunteers whose subsequent clinical and serological progress was carefully monitored. ${ }^{22}$ The study concluded that early treatment of syphilis probably prevented the development of any immunity to the organism, but that pretreatment with heat-killed organisms significantly reduced subsequent reinfection with live organisms. Such an anamnestic response could not be correlated to titres of antibody against $T$ pallidum and seemed to relate more closely to cell-mediated immune functioning.

Recently, emphasis has been placed on the in-vitro response of lymphocytes to improved preparations of syphilitic antigens. ${ }^{121323-26}$ Most of these studies measured a lymphoblastic response by morphological criteria rather than by measuring DNA synthesis; however, in general they agree that sensitised lymphocytes are present and can respond to syphilitic antigens in late primary disease and persist from that time. These studies require confirmation with more sensitive techniques.

If we can accept that most subjects can develop a cellular immune response to these antigens, clearly the clinical course of some patients with syphilis suggests that an inadequate immune response to $T$ pallidum can occur early in the disease. Our data do not suggest that the lymphocytes from patients with primary or secondary disease respond abnormally to either PHA or allogeneic lymphocytes. Clearly, however, sera from some primary and many secondary cases of syphilis are capable of reducing the response of these cells and normal cells to both PHA and allogeneic lymphocytes. It remains to be shown that the presence of these factors in vivo is associated with a less favourable clinical course.

Previous data from this laboratory ${ }^{14}$ have pointed out the difficulty of assessing immunosuppressive properties of individual sera. Normal lymphocytes respond reliably to both PHA and allogeneic 
lymphocytes in autologous serum but the variation of response in normal but heterologous sera exceeds two logs. This range can be considerably reduced by using pooled human plasma. From the present studies we suggest that an active immunosuppressive property of a serum sample, as opposed to a passive nonsupportive property, can only be proved when cells in culture are supplied with optimal amounts of pooled human plasma before the test serum is added. A suppression of the response in these circumstances is likely to be biologically, although not necessarily clinically, significant.

Although it is difficult to assign a degree of responsiveness to PHA and allogeneic lymphocytes that correlates with adequate in-vivo CMI function, it seems likely that the degree of real suppression noted here would still leave most lymphocytes responding within the lower limits of response encountered in healthy individuals. A weak immunosuppressive effect of serum could result from dilution in the plasma volume of an agent exerting a much more powerful effect at the tissue level. The effect of serum from cases of primary and secondary disease on the responsiveness of sensitised lymphocytes to treponemal antigens would provide more relevant information but would require a more sensitive invitro assay than has so far been described. It has been suggested ${ }^{27}$ and shown $^{28}$ that the presentation of large amounts of antigen to sensitised lymphocytes can produce a transient, generalised suppression of CMI; this suppression ends once high-zone tolerance, specific for the desensitising antigen, develops. Such a non-specific humoral immunosuppressive factor may be the product of suppressor $T$ cells. ${ }^{27}$ In patients with late primary and early secondary disease, when numerous foci of infection are present, the conditions necessary for eliciting such factors-which could be detected in serum by the methods detailed here-may exist. Perhaps a progression to true immunological tolerance to treponemal antigens occurs in many cases of late syphilis. Once tolerance developed, non-specific humoral immunosuppressive factors may disappear.

The immunostimulatory nature of the sera from cases of congenital syphilis is definite but unexplained. In view of the histological data showing decreased numbers of lymphocytes in the spleen of children with this disease ${ }^{8}$ and the fact that the spleen is a major source of suppressor $T$ cells, it seems reasonable to suggest that congenital infection may lead to a lack of suppressor $T$ cells and so to a lack of the humoral regulation of CMI responses which they usually provide. Sera containing helper factors but not regulatory factors would appear to be immunostimulatory. If this explanation was correct, then other phenomena associated with defective T-cell regulatory functions-such as the incidence of autoimmune reactions-would be increased in such patients. The literature contains no information on this matter. Clinical pressures demand more research into these questions; with improved technology, we are studying separated effector and suppressor $T$ cells in humans.

Support for this work was given by NIH training grant 00271 and NIH grant AI-11785.

\section{References}

1. Bullock WW, Katz DH, Benacerraf B. Induction of $T$ lymphocyte responses to a small molecular weight antigen. III $\mathrm{T}$-T interaction to determinants linked together: suppression vs enhancement. J Exp Med 1975; 142:261-74.

2. Feigheny C, Whelan CA, Weir DG, Greally JF. In-vitro studies of suppressor cell function in human peripheral blood mononuclear cells. Clin Exp Immunol 1978; 32:459-65.

3. Gershon RK. A disquisition on suppressor T cells. Transplant $\operatorname{Rev} 1$ 1975; 26: 170-85.

4. Dwyer JM, Mangi RJ, Gee B, Kantor FS. Comparison of the anergy of sarcoidosis with experimentally induced anergy in guinea pigs. Annals NY Acad Sci 1976;278:29-35.

5. Hillinger SM, Herzig GP. Impaired cell-mediated immunity in Hodgkin's disease mediated by suppressor lymphocytes and monocytes. J Clin Invest 1977; 59: 1620-7.

6. Jayawardena AN, Waksman BH. Suppressor cells in experimental trypanosomiasis. Nature 1977; 265:539-41.

7. Levene GM, Wright DJM, Turk JL. Cell-mediated immunity and lymphocyte transformation in syphilis. Proc Royal Soc of Med 1971;64: 14-8.

8. Levene GM, Turk JL, Wright DJM, Grimble AGS. Reduced lymphocyte transformation due to a plasma factor in patients with active syphilis. Lancet 1969; ii:246-7.

9. From E, Thestrup-Pedersen K, Thulin H. Reactivity of lymphocytes from patients with syphilis towards $T$ pallidum antigen in the leucocyte migration and lymphocyte transformation tests. Br J Vener Dis 1976; 52:224-9.

10. Wright DJM, Grimble AGS. Why is the infectious stage of syphilis prolonged? Br J Vener Dis 1974; 50:45-9.

11. Musher DM, Schell RF, Jones RH, Jones AM. Lymphocyte transformation in syphilis: an in-vitro correlate of immune suppression in vivo? Infect Immun 1975; 11:1261-4.

12. Badanoiu A, Gavrilesco $\mathbf{M}$, Nicolau G, Circiumaresco $T$. Lymphoblastogenese immunoallergique et antigène treponemique in vitro dans la syphilis. Roum Path Exp Microbiol 1969; 28: 1901-6.

13. Janot C, Grandidier M, Pupil P, Thomas J-L, Beurey J, de Lavergne $\mathrm{E}$. Le test de transformation lymphoblastique au cours de la syphilis. Presse Medicale 1971;79:1901-4.

14. Mangi RJ, Dwyer JM, Kantor FS. The effect of plasma upon lymphocyte response in vitro. Demonstration of a humoral inhibitor in patients with sarcoidosis. Clin Exp Immunol 1974; 18:519-28.

15. Schaudinn F, Hoffman E. Arb Kais Gesund 1905;22:528.

16. Neisser A. Arb Kais Gesund, 1911; 37:201.

17. Noguchi, H. J Exp Med 1911;14:557.

18. Chesney, AM. Immunity in syphilis. Medicine Monographs XII Baltimore; Williams and Wilkins, 1972.

19. Marshak LC, Rothman S. Skin testing with a purified suspension of $T$ pallidum. Amer $J$ Syphilis 1951;35:35-41.

20. Thivolet J, Simeray A, Rolland $\mathbf{M}$, Challut F. Etude de l'intradermoreaction aux suspensions de treponèmes formolées (Souche Nichols Pathogene) chez les syphilitiques et les sujets normaux. Annales de l'Institut Pasteur 1953;83:23-33

21. Laird SM, Thorburn AL. Assessment of the "Luotest" in late syphilis. Br J Vener Dis 1966; 42:119-21.

22. Magnuson HJ, Thomas EW, Olansky S, Kaplan BJ, DeMello $\mathrm{L}$, Cutler JC. Inoculation syphilis in human volunteers. Medicine 1956; 35: 33-82.

23. Chieregato GC, Faldarini G, Pagnes P. La stimolazione linfocitaria. In vitro in sogetti luetici. Minerva Dermatol 1967;42:406-7. 
24. Dwyer JM, Mackay IR. Modern concepts of antigen binding receptors on lymphocytes. Their nature and role in immune responses. Rev Europa Etudes Clinique et Biol 1971; 16: 743-6.

25. Beurey J, Weber M, Janot C, Grandidier M, Pupil P. L'allergie au cours de la syphilis. Interet du test de transformation lymphoblastique. Ann Dermatol Syphilol 1971;98:536-8.

26. Basset A, Badanoiu A, Grosshans E, et al. Studio sulla reattivita immuno-cellare nella lue con il test della transformazione blastica dei linfociti in vitro (TTL). Minerva Medica 1972;63:3031-6.
27. Gershon RK. T-cell control of antibody production. Contemporary Topics in Immunobiology. Vol 3, chap 1, New York: Plenum Press, 1974.

28. Dwyer JM, Kantor FS. In-vivo suppression of delayed hypersensitivity: Prolongation of desensitization in guinea pigs. J Exp Med 1975; 142: 588-99. 